\title{
LA EVALUACIÓN PSICOLÓGICA DE LAS PREOCUPACIONES HIPOCONDRÍACAS
}

\author{
Ma Pilar Martínez Narváez-Cabeza de Vaca' \\ Amparo Belloch Fuster ${ }^{1}$ \\ Cristina Botella Arbona ${ }^{2}$ \\ 1 Universitat de Valencia \\ 2 Universitat Jaume I, Castellón.
}

\begin{abstract}
RESUMEN
En los últimos años se ha producido un aumento del interés por el estudio de la hipocondría, tal y como se revela en el aumento de publicaciones referidas a este trastorno. Paralelamente, se han producido progresos significativos en diversos ámbitos, entre ellos, el que hace referencia a la evaluación psicológica del mismo. En el presente trabajo se recogen las indicaciones más relevantes, en opinión de las autoras, para llevar a cabo la evaluación psicológica de la hipocondría: pautas para la entrevista de evaluación y principales instrumentos de valoración. Finalmente, se sugieren algunos aspectos a considerar en los futuros trabajos de investigación sobre la evaluación de las preocupaciones hipocondriacas.
\end{abstract}

Palabras clave: hipocondría, evaluación psicológica, entrevista, instrumentos psicológicos.

\begin{abstract}
The interest in the study of hypochondriasis has been increased in the last few years, such as is revealed in the number of publications about the disorder. Subsequently, there has been a remarkable progress in the knowledge of this psychopathological condition, as well in their psychological assessment. This paper includes the main components to be considered to the psychological assessment of hypochondriasis: the guidelines to the assessment interview and the principal measure instruments. Finally, some suggestions for future research about the assessment of hypochondriacal preoccupations are offered.
\end{abstract}

Key words: hypochondriasis, psychological assessment, interview, instruments 


\section{INTRODUCCIÓN}

Los pacientes hipocondriacos y/o somatizadores constituyen un porcentaje importante de los usuarios de los servicios públicos de salud. De hecho, una parte considerable de los recursos sanitarios se dedica a dar respuesta a esta demanda. La repercusión que conlleva para la red sanitaria la no identificación o el trato inadecuado de este tipo de pacientes, se deja sentir básicamente a dos niveles. En primer lugar, estos pacientes perturban la dinámica de funcionamiento al acaparar una parte sustancial de los medios humanos y técnicos disponibles a través de consultas médicas recurrentes, ingresos hospitalarios, uso o demanda injustificada de pruebas clínicas, etc. En segundo lugar, y consecuentemente con lo anterior, consumen una porción importante de los presupuestos económicos destinados a cubrir los gastos de sanidad.

Dadas las consecuencias que puede ocasionar la hipocondría -y los problemas de somatización en general- en el sistema público sanitario, amén de las que genera a la propia persona que la padece (p. ej., malestar emocional), no resulta sorprendente que en las últimas décadas se hayan elaborado diversos instrumentos para la detección eficaz de este tipo de trastomo. Una de las primeras medidas utilizadas fue la escala de Hipocondriasis del Inventario de Personalidad Multifásico de Minnesota (MMPI) construida por Hathaway y MacKinley en 1943. Varias décadas más tarde, se confeccionaron los dos instrumentos más utilizados para la evaluación de la hipocondría y la conducta anormal de enfermedad: el Cuestionario de Conducta de Enfermedad (IIIness Behaviour Questionnaire, IBQ) de Pilowsky y Spence (1983), y las Escalas de Actitud hacia la Enfermedad (Illness Attitude Scales, IAS) de Kellner (1986). A partir de la publicación de la tercera versión del Manual Diagnóstico y Estadístico de los Trastomos Mentales, DSM-III (APA, 1980) y sus revisiones posteriores DSM-III-R y DSMIV (APA, 1987,1994), empezaron a diseñarse entrevistas diagnósticas para la identificación de la hipocondria de acuerdo con los criterios diagnósticos contemplados en estos manuales. Así, aparecen las Entrevistas Clínicas Estructuradas de Spitzer, Williams, Gibbon y First (1990) y de Barsky, Cleary, Wyshak, Spitzer, Williams y Klerman (1992). Asimismo, durante la década de los 90 el interés de la evaluación se ha centrado en elaborar instrumentos para la identificación de subtipos de hipocondría tales como la fobia a la enfermedad (p. ej., Escala de Preocupaciones por la Enfermedad -Illness Concerns Scale, ICS- de Wesner y Noyes, 1991); modalidades específicas de temores hipocondriacos tales como la fobia cardiaca (p. ej.. Cuestionario de Ansiedad Cardiaca -Cardiac Anxiety Questionnaire, CAQ- de Eifert, Seville, Brown, Anthony y Barlow, 1992); y procesos cognitivo-perceptivos involucrados en el desarrollo de los problemas hipocondriacos tales como la tendencia a amplificar los síntomas corporales (p. ej., Escala de Amplificación Somatosensorial -Somatosensory Amplification Scale, SSAS- de Barsky, Wyshak y Klerman, 1990).

Por otra parte, en los últimos años también se ha prestado gran atención al estudio de las propiedades que debe poseer, tanto en términos de forma como de contenido, la entrevista de evaluación con pacientes que presentan preocupaciones hipocondriacas. Entre los autores que más han analizado este aspecto destacan Salkovskis y Warwick, quienes han senalado, como aspectos básicos a contemplar en la entrevista, la diferenciación entre la hipocondría y otros posibles trastornos, la exploración de las características del problema, y la resolución de los conflictos que pueden surgir en la relación terapéutica.

El presente trabajo tiene como finalidad describir la evaluación de la hipocondria. Para ello, y atendiendo al estado actual de conocimientos en este ámbito, se plantean las pautas más adecuadas a seguir durante la entrevista de evaluación con el paciente, asi como los cuestionarios de auto-informe estandarizados a utilizar para la valoración de la sintomatologia.

\section{guía PARA LA ENTREVISTA DE EVALUACIÓN}

La entrevista de evaluación de un paciente que presenta preocupaciones desmesuradas por su salud persigue tres objetivos (Wanwick, 1995). El primero de ellos consiste en verificar que el paciente padece un trastomo hipocondriaco y no otro tipo de desorden físico o psicológico. El segundo es el de elaborar una explicación detallada de la psicopatología del paciente, de tal manera que se pueda realizar una formulación 
psicológica apropiada de su problema. Además, hay que cerciorarse de que el tratamiento cognitivo-comportamental que se pretende aplicar es el idóneo para el paciente al que va destinado. El tercer objetivo estriba en establecer una adecuada relación terapéutica. Este objetivo no es, desde luego, específico de la hipocondria, pero reviste una especial importancia con este grupo de personas. Por ello, resulta del máximo interés procurar que el paciente perciba la evaluación como satisfactoria y completa y al terapeuta como empático y competente, lo que facilitará que acepte el tratamiento propuesto.

Partiendo de este triple objetivo y basándonos en las aportaciones de Salkovskis (1989) y Warwick (1995), se plantean a continuación un conjunto de directrices generales que pueden servir como gula para la entrevista de evaluación de la hipocondria.

\section{Diagnóstico diferencial}

El primer paso de la evaluación consiste en realizar un correcto diagnóstico diferencial confirmando que la psicopatologia que presenta el paciente se corresponde con un trastomo hipocondriaco (Warwick, 1995). Existen algunas condiciones clínicas que, al cursar con sintomas fisicos, pueden confundirse con la hipocondria y a los que el clínico debe prestar suma atención para evitar diagnósticos erróneos (Chorot y Martínez, 1995; Martínez, 1997). Puesto que la preocupación excesiva por la salud no es un antidoto contra la enfermedad, el primer paso en el diagnóstico diferencial es excluir la existencia de un trastorno físico. Algunas enfermedades orgánicas se acompañan de quejas somáticas múltiples $e$ inespecificas que, al inicio de su evolución, pueden simular o emular sintomas somatoformes. Entre los problemas orgánicos cuya presencia hay que descartar figuran los siguientes (APA, 1994; Marshall, 1987): trastornos endocrinos ( $p$. ej., enfermedades tiroideas o paratiroideas), esclerosis múltiple, miastenia grave, lupus eritematoso sistémico, anemia perniciosa, tumores, enfermedad coronaria atipica y sindromes virales crónicos ( $p$. ej., hepatitis crónica).

Ahora bien, aunque esté presente una enfermedad orgánica que justifique la sintomatologia fisica, debe considerarse un aspecto crucial en el diagnóstico diferencial de la hipocondria, pues también es cierto que ambas condiciones pueden coexistir. AsI, cuando esté presente un trastorno orgánico es posible establecer un diagnóstico de hipocondrla en los cuatro casos siguientes (Schmidt, 1994): 1) el sujeto interpreta catastróficamente la información médica acerca de la enfermedad; 2) no se tranquiliza cuando el médico le confirma el pronóstico favorable de la enfermedad; 3) su temor o creencia se basa en síntomas físicos independientes de la enfermedad; y 4) las preocupaciones hipocondriacas son previas a la aparición de la enfermedad.

También hay que descartar la presencia de otras psicopatologías tales como los trastornos del estado de ánimo (p. ej., depresión) y los trastomos de ansiedad (p. ej., trastomo por angustia, trastomo por ansiedad generalizada y trastorno obsesivo-compulsivo), que pueden presentar caracteristicas clínicas similares a las de la hipocondría (Chorot y Martinez, 1995; Martínez, 1997). En cuanto a los primeros, Dubovsky (1988) ha señalado algunos elementos que ayudan a diferenciar entre la sintomatologia asociada a un estado de ánimo depresivo y los trastornos somatoformes. Asi, apunta que en la depresión, a diferencia de los trastornos somatoformes, existe un predominio de la sintomatologia vegetativa, que se suele agravar por la mañana; una historia previa y familiar de depresión y trastomos relacionados; una pérdida temprana, tras la cual aparecen los sintomas; una tendencia por parte del paciente a separarse de los médicos y de las personas significativas, a minimizar $u$ ocultar los sintomas y a aceptar el origen psicológico de sus síntomas; y una respuesta positiva a los antidepresivos.

Respecto a los trastornos de ansiedad, Salkovskis y Clark (1993) han senalado que ol trastorno por angustia, a diferencia de la hipocondria, se caracteriza por: 1) interpretación errónea como signo de enfermedad de sintomas somáticos asociados a la activación fisiologica; 2) atribución de tales sintomas corporales a una grave enfermedad que está ocurriendo ya o está a punto de suceder; 3) conductas de evitación, escape y búsqueda de seguridad; y 4) creencias disfuncionales sobre la ansiedad. Barsky (1992a) ha subrayado una serie de diferencias entre la fenomenología del trastorno hipocondriaco y del obsesivo-compulsivo. A nivel 
cognitivo ambas condiciones difieren en que en esta última existen ideas que interrumpen el curso del pensamiento, que el sujeto considera absurdas $y$, por ello, trata de eliminarlas. También difieren ambas condiciones a nivel interpersonal y fisico, en la medida en que en el trastorno obsesivo-compulsivo el sujeto procura ocultar su problema y están ausentes las sensaciones somáticas, o resultan poco significativas clinicamente. Por otra parte, también se aprecian algunas diferencias entre los dos trastomos centradas en la actitud del paciente ante las conductas que exhibe: el obsesivo-compulsivo realiza intentos por resistirse a ellas puesto que estima que son excesivas, al menos al inicio del trastorno. En cuanto al trastorno por ansiedad generalizada, Woody y Rachman (1994) han señalado los siguientes puntos de divergencia respecto a la hipocondria: 1) el temor a la enfermedad se focaliza en alguna persona querida; 2) la amenaza está orientada al futuro; 3) resulta más dificil lograr tranquilizar al paciente mediante la información médica; y 4) los temores no requieren activadores o precipitantes.

\section{Contenido de la evaluación}

Con la entrevista de evaluación se pretende profundizar en el conocimiento del problema del paciente. Generalmente, esto se consigue gracias a la información verbal que él mismo proporciona. Además, es posible completar la información utilizando una anamnesis clínica y un cuestionario biográfico. Como apunta Warwick (1995), en algunas ocasiones puede ser conveniente ampliar nuestro conocimiento sobre el problema del paciente a través de su historial médico, los juicios clínicos emitidos por los profesionales de la salud que lo han atendido, y los datos aportados por los familiares.

Las cuestiones más relevantes a explorar en la entrevista de evaluación con el paciente son las siguientes (Salkovskis, 1989; Warwick, 1995):

1. Actitud del paciente hacia el tratamiento psicologico. Examinar las creencias y expectativas del paciente con respecto a la terapia y a los posibles resultados que puedan derivarse de ésta con preguntas tales como: ¿cree que seguir un tratamiento psicológico puede ayudarle?.

2. Descripción breve del problema. Solicitar al paciente que describa de manera resumida su problema actual. Para ello se puede recurrir a preguntas del siguiente tipo: “¿puede decirme en pocas palabras cuál es su problema?". Asimismo, para ilustrar las características generales del problema, suele ser útil que el paciente proporcione ejemplos de ocasiones recientes en las que se ha preocupado mucho por su salud y que narre cómo se desarrolla un día típico de su vida.

3. Historia del problema. Identificar los factores responsables del inicio del problema, de la fluctuación en la gravedad de los síntomas, de la persistencia de la preocupación, etc. Preguntas como las siguientes pueden servir para explorar esta clase de datos: "¿cuándo empezó a preocuparse por su salud del modo en que lo hace ahora?", "¿en qué circunstancias?", "¿la preocupación por su salud ha ido aumentando progresivamente?", etc.

4. Descripción detallada del problema. Obtención de información específica sobre el estado del paciente, centrada en la exploración de los componentes fisiologicos, cognitivos y conductuales implicados en el problema. Los aspectos esenciales de estos componentes que hay que explorar y los procedimientos a utilizar para ello son los siguientes:

a) Componente cognitivo. Incluye los pensamientos negativos automáticos sobre el significado de los sintomas, las imágenes mentales sobre la enfermedad y la muerte, los factores cognitivos que mantienen la ansiedad por la salud ( $p$. ej., focalización de la atención en el cuerpo) y los supuestos disfuncionales acerca de la salud y de la enfermedad. Para recabar esta información se recurre al tipo de preguntas que se utilizan en la terapia cognitiva (p. ej.. "cuando empezó a sentir los sintomas, ¿qué pensamientos pasaron por su mente?"); a la visualización (en imaginación) y a la descripción de episodios recientes de ansiedad por la salud (p. ej., pedir al paciente que trate de imaginar y revivir un momento de preocupación y que describa lo que estaba pensando, sintiendo, haciendo, etc.); y a los registros diarios de episodios de preocupación en los que el paciente anota los sintomas corporales molestos y la estimación de la gravedad de éstos, la hora del dia y situación antecedente en la que se experimentan, las atribuciones sobre la posible causa que los origina y evaluación del grado de creencia en tales atribuciones, las emociones que 
provocan, su intensidad, y las consecuencias conductuales que desencadenan. Asimismo, para la exploración de los supuestos disfuncionales sobre la salud/enfermedad se utiliza el método de la flecha descendente, que consiste en la formulación de preguntas que se repiten sucesivamente hasta llegar a un enunciado suficientemente general como para incluir la situación problemática actual y otras de naturaleza similar (p. ej.: "imagine que to que piensa es verdad, ¿qué implicaria?").

b) Componente conductual. Incluye las conductas que desencadenan la preocupación por la salud, las que participan en su mantenimiento, y las consecuencias derivadas de éstas. Para explorar este componente se realizan preguntas que permitan, por ejemplo, identificar las conductas de verificación corporal (p. ej., "¿se toca para ver qué nota o siente en su cuerpo?"), evitación de situaciones o actividades (p. ej., "¿hay algo que no pueda hacer debido a la preocupación por su salud?"), y búsqueda de información tranquilizadora ( $p$. $\theta$ j. "¿trata de informarse sobre la enfermedad que le preocupa?"). También se puede hacer uso de los registros diarios.

c) Componente fisiológico. Incluye las sensaciones corporales que son interpretadas erróneamente, los sintomas fisicos derivados de la activación del Sistema Nervioso Autónomo que aparecen durante los episodios de preocupación, y los cambios corporales que surgen como consecuencia de las conductas de comprobación corporal. Para detectar la localización, intensidad, frecuencia, etc. de estos sintomas se emplean los registros diarios.

5. Variables moduladoras. Identificación de los factores situacionales, conductuales, cognitivos, afectivos, interpersonales y fisiológicos que hacen que el problema empeore (p. ej. " $¿ e n$ qué momentos es más probable que aparezca la preocupación por su salud o que se intensifique?") y de los que consiguen mejorarlo (p. ej. "¿hay algo que le ayude a controlar su problema?").

6. Consecuencias del problema. Evaluación del grado en que el problema perturba la vida del paciente a nivel social (p. ej., "¿en qué medida afecta la preocupación por su salud a sus relaciones sociales habituales?"), laboral (p. ej. "¿su problema influye en su desempeño en el trabajo?"), ocio (p. ej. "¿la preocupación por su salud dificulta las actividades que realiza en su tiempo libre?"), etcétera.

7. Historia de tratamientos anteriores. Exploración de las consultas y revisiones médicas realizadas, los resultados obtenidos y la atribución de la mejoría o el empeoramiento. Las preguntas siguientes pueden servir como referencia: "¿ha consultado su problema con algún médico?, ¿con cuántos?, ¿qué pruebas clínicas le han hecho?, ¿cuáles fueron los resultados?".

8. Episodios previos de preocupación por la salud. Identificación de momentos anteriores al episodio actual en los que el paciente se ha inquietado de manera desmedida por su estado físico ( $p$. ej., "¿ha habido alguna temporada previa a la actual en la que se haya preocupado mucho por su salud?").

9. Historia personal y familiar de enfermedades. Detección en la vida del paciente o de sus familiares de hechos relevantes relacionados con la enfermedad. Algunas de estas preguntas pueden ayudar a recabar esta información: por ejemplo, "¿qué enfermedades importantes ha padecido?", "¿hay o ha habido algún caso de enfermedad grave en su familia?".

Además de rastrear el problema a través de preguntas sobre estas cuestiones, con frecuencia es aconsejable obtener datos adicionales mediante cuestionarios de autoinforme estandarizados (más adelante, en el eplgrafe dedicado a los instrumentos de evaluación se comentan algunos de los más relevantes) y pruebas comportamentales (p. ej., ejercicios de hiperventilación).

\section{Relación terapéutica}

Resulta bastante usual que los pacientes hipocondriacos se muestren insatisfechos con las consultas médicas. Según Warwick (1995), sus principales quejas sobre el trato que reciben por parte de los médicos así como la manera en que el terapeuta puede resolverlas son las siguientes:

1. Sensación de no haber recibido una explicación satisfactoria de sus problemas. En muchos pacientes las preocupaciones por la salud tienen varios años de evolución, a lo largo de los cuales han consultado a varios médicos. Con frecuencia éstos se han limitado a decir que "no les pasa nada malo", pese a lo cual los pacientes insisten en conocer la causa de 
su malestar. Ante esta situación el terapeuta ha de explicar al paciente que uno de los objetivos de la evaluación consiste en proporcionarle una formulación psicológica adecuada del problema sobre la que se estructurará el tratamiento. Además, ha de comprobar que el paciente ha comprendido bien la explicación ofrecida, y ha de solventar las dudas que este pueda tener al respecto.

2. Sensación de no haber tenido la oportunidad de explicar sus problemas adecuadamente $y$ en su totalidad. En la situación de consulta el paciente suele estar tan nervioso que llega a distorsionar la información que le da el facultativo. Además, tras su visita al médico suele "rumiar" sobre lo dicho en la consulta, examinar las palabras y gestos del médico tratando de descubrir significados ocultos, etc. La actuación del terapeuta en este punto ha de estar centrada en comunicar al paciente estas dificultades y en hacerle saber que puede interrumpirle en cualquier momento para preguntar 0 añadir datos complementarios. Asimismo, ha de verificar que el cliente ha entendido correctamente los temas tratados, y ha de intentar resolver cualquier mal entendido, insistiendo en que toda preocupación adicional que pueda surgir será abordada en la próxima consulta.

3. Descontento por la actitud de los profesionales de la salud hacia sus problemas. A menudo el paciente siente que no recibe un trato correcto por parte del médico y tiene la impresión de que éste le considera un pesado o alguien que finge sus sintomas. El paciente suele anticipar esta reacción de los médicos, lo que le conduce a interpretar mal la información y a confirmar asi sus expectativas. El terapeuta puede compensar este descontento del paciente mostrando un estilo empático, e incluso discutiendo abiertamente, cuando la situación lo requiera, los sentimientos del paciente. También resulta de ayuda insistir en que los sintomas somáticos son considerados como reales (no como imaginarios), y no sorprenderse por las descripciones que el paciente pueda hacer de su problema (p. ej., miedos extraños o poco comprensibles). A diferencia de la fase de tratamiento en la que puede hacerse uso del humor, en la fase de evaluación no es recomendable recurrir a éste por los recelos que puede generar en el paciente.
4. Discrepancias entre su agenda y la del terapeuta que provoca insatisfacción con la consulta. El paciente tiende a percibir la evaluación y el tratamiento psicológico como un obstáculo para someterse a exploraciones médicas; en cambio, el terapeuta considera que es precisamente el tratamiento psicológico lo que necesita el paciente. Este conflicto puede resolverse discutiendo abiertamente ambas posturas $y$ mostrando comprensión hacia la actitud del paciente, lo que facilitará que éste acepte la conveniencia de abordar su problema desde una perspectiva psicologica.

\section{INSTRUMENTOS DE EVALUACIÓN}

\section{Evaluación categorial}

El establecimiento de un diagnóstico categorial de hipocondria ( $y$ en general de cualquier otro trastorno psicológico) según las pautas establecidas en el DSM o la CIE tiene una serie de ventajas, pero también ciertas desventajas. Según Lorr (1986), entre las ventajas de los modelos categoriales cabe destacar las siguientes: 1) favorecen la comunicación o la creación de diseños de investigación; 2) son fáciles de memorizar; y 3) permiten dar una mejor respuesta a las demandas de una organización institucional asistencial. A estos aspectos positivos podrian anadirse dos más (Lemos, 1994): confieren unidad a la sintomatología que manifiesta una persona $y$, además, constituyen estándares de referencia consolidados entre los clínicos. Las desventajas que conllevan los modelos categoriales se refieren, en primer lugar, al hecho de que contribuyen a mantener la errónea creencia de que los procesos psicopatológicos constituyen entidades discretas frente a la consideración de que únicamente son conceptos que facilitan la coordinación de nuestras observaciones. La segunda limitación consiste en la pérdida de información que acarrean, ya que las categorias dan prioridad a una o dos características excluyendo otros posibles aspectos relevantes. El último aspecto negativo se refiere a que el número y variedad de las categorías resulta insuficiente para dar cuenta de la diversidad individual que se observa en la práctica clinica (Millon, 1991).

Al margen de las "virtudes" y "defectos" atribuibles a los sistemas clasificatorios categoriales, cuando se estima conveniente estable- 
cer un diagnóstico atendiendo a los criterios del DSM o de la CIE, resulta muy útil recurrir a entrevistas estructuradas. Este tipo de instrumento permite hacer un recorrido metódico por los criterios diagnósticos definidos para el trastomo, utilizando una pauta estandarizada de preguntas y un sistema de valoración de las respuestas emitidas por el sujeto. A continuación se comentan las entrevistas clínicas estructuradas más utilizadas para la identificación de la hipocondria.

\section{Entrevista Clinica Estructurada para el DSM-} III-R (Structured CIInical Interview for DSMIII-R; Spitzer, WIIlams, Gibbon y First, 1990)

Esta entrevista permite establecer un diagnóstico de la hipocondría de acuerdo con los criterios del DSM-III-R (APA, 1987). Contiene una serie de preguntas específicas que exploran el cumplimiento de dichos criterios, una breve gula orientativa para el entrevistador y un código para calificar las respuestas del sujeto ( $?=$ información insuficiente; 1 = ausente o falso; 2 = subumbral; y 3 = umbral o verdadero).

\section{Entrevista Diagnóstica Estructurada para la} Hipocondria (Structured Dlagnostic Interview for Hypochondriasis; Barsky, Cleary, Wyshak, Spltzer, Willams y Klerman, 1992)

Este instrumento permite obtener un diagnóstico DSM-III (APA, 1980) de la hipocondría, a través de una serie de preguntas detalladas que exploran exhaustivamente los criterios de dicho sistema diagnóstico. La valoración de las respuestas del sujeto a las preguntas formuladas es dicotómica ("si" o "no"). Esta entrevista ha mostrado elevada fiabilidad interjueces, buena validez concurrente con el Indice Whiteley y buen poder discriminante (Avia, 1993). En nuestras investigaciones hemos introducido algunas modificaciones en este instrumento con el propósito de abarcar los criterios diagnósticos introducidos en las posteriores ediciones del Manual (DSM-III-R y DSM-IV).

LIsta de Comprobaclón de Sintomas (Symptom Checkllst; Janca, Ustün y van Drimmelen, 1992)

Se trata de una gula diagnóstica clínica que facilita la codificación de los trastornos psico- lógicos (entre ellos los trastomos somatomorfos) en función de las diferentes pautas diagnósticas de la CIE-10 (OMS, 1992). Esta guia contiene una serie de Items que describen brevemente las caracteristicas clínicas sobre las que se fundamenta el diagnóstico, y a los que el clínico ha de contestar en términos de ausencia o presencia de la característica explorada.

\section{Evaluación dímensional}

Frente a la conceptualización de los trastornos mentales que realizan los modelos categoriales que subyacen a los sistemas clasificatorios como el DSM o la CIE, se han propuesto diversos planteamientos dimensionales para describir la sintomatología psicológica. Al igual que sucedia con el enfoque categorial, la adopción de una perspectiva dimensional de los trastornos mentales comporta una serie de ventajas $\theta$ inconvenientes. Entre los aspectos positivos Lemos (1994) ha recopilado los siguientes: 1) permite establecer diversas combinaciones de características clínicas en una representación multifactorial que minimiza la pérdida de información; 2) facilita la clasificación de los casos atípicos; 3) favorece la conceptualización de la anormalidad y la normalidad como extremos de un continuo; y 4) facilita la detección de los cambios experimentados por los sujetos. Sin embargo, este enfoque también presenta ciertas limitaciones (APA, 1994): 1) las descripciones dimensionales son menos familiares y vivas que las denominaciones de las categorias diagnósticas; y 2) no existe acuerdo acerca de cuáles son las dimensiones básicas que deben contemplarse para representar los problemas psicológicos. A estos problemas podría sumarse otro, más referido a la falta de acuerdo en cuanto al significado del término "dimensión", ya que como recogen Clark, Watson y Reynolds (1995), este vocablo ha sido utilizado para hacer referencia a cuestiones tan dispares como niveles de gravedad de grupos de sintomas, síntomas fenotípicos o rasgos de personalidad que se combinan de diversas formas para dar lugar a distintas psicopatologias, factores de vulnerabilidad genética subyacentes, y parámetros biológicos especificos.

Prescindiendo de estas limitaciones, cabe decir que la hipocondria, además de ser entendida como una entidad nosológica, esto es, 
como un problema que existe en la población de forma discontinua y que puede estar presento 0 ausente, también puede ser considerada como una dimensión psicopatológica (continuo lineal de gravedad) que se da en mayor o menor medida en diversos trastornos (tanto físicos como psiquicos) y que es evaluable en cualquier persona. Asi pues, y utilizando las palabras de Barsky, Wyshak y Klerman (1986), "el hipocondríaco (...) puede ser al estoico, como el fanático religioso lo es al ateo, o el fanático político lo es al ciudadano politicamente indiferente" (p. 493). En los últimos años se ha venido defendido que las preocupaciones por la salud que todo ser humano experimenta en algún momento de su vida, son comparables a las que se observan en los estados hipocondríacos clínicos, es decir, la diferencia entre ambas es únicamente una cuestión de grado.

Algunos autores argumentan que los trastornos somatoformes no constituyen categorias diagnósticas discretas sino que forman parte de la conducta anormal de enfermedad, entendida como una dimensión. Asl, por ejemplo, Ford (1983) defiende la existencia de dos continuos, uno caracterizado por un estilo cognitivo obsesivo en el que se incluiria la hipocondria; y otro caracterizado por un estilo cognitivo histérico que englobaria el trastorno por somatización, los trastornos facticios y el sindrome de Munchausen (Ford y Parker, 1990). Costa y McCrae (1985) han propuesto un modelo dimensional de las preocupaciones somáticas para dar cuenta de la relación entre las condiciones médicas (salud objetiva) y las quejas somáticas (salud subjetiva). Consideran que existen diferencias consistentes y persistentes entre los individuos en cuanto a la percepción, interpretación e informe de sintomas fisicos, y que cada uno de nosotros podría ubicarse en algún punto a lo largo de un continuo, que comprendería desde la escasa comunicación de sintomas hasta la hipocondría. Salkovskis y Warwick (1986) también han defendido un planteamiento dimensional de la hipocondrla. Estos autores proponen la existencia de un continuo de preocupación por la salud que se extenderia desde las preocupaciones leves por alguna sensación física inusual, hasta las preocupaciones y miedos por los sintomas corporales en los que todos los pensamientos y actividades giran en torno a la enfermedad. En la línea de los planteamientos anteriores, Kirmayer, Robbins y Paris (1994) entienden la somatización como un constructo dimensional, que permite situar los distintos casos psicopatológicos a lo largo de un continuo con diversos grados de perturbación. Estos autores distinguen tres constructos dimensionales de somatización: tendencia a experimentar $\theta$ informar sintomas funcionales, tendencia a preocuparse o creer que se está enfermo, y tendencia de algunas personas con problemas de ansiedad o depresión, a presentar un cuadro clínico en el que predomina la sintomatologia somática.

Aunque estos supuestos de continuidad no son todavía una realidad demostrada, si han recibido apoyo de algunos estudios como, por ejemplo, los temores hipocondríacos de los estudiantes de medicina (Kellner, Wiggins y Pathak, 1986) y la preocupación excesiva por la salud de las mujeres gestantes (Fava, Grandi, Michelacci, Saviotti, Conti, Bovicelli, Trombini y Orlandi, 1990), entre otros.

Con respecto al ámbito clínico aplicado, en la evaluación de un paciente que muestra preocupaciones de carácter hipocondriaco, además de efectuar el diagnóstico del trastomo atendiendo a los criterios diagnósticos de un sistema clasificatorio como el DSM-IV o la CIE10 , es recomendable realizar un análisis cuantitativo de la gravedad de los sintomas, que permita conocer el curso del trastorno y el grado de cambio terapéutico. Con tal propósito se han creado diversos instrumentos de autoinforme. A continuación se describen los que se utilizan con mayor frecuencia y que presentan mejores caracteristicas psicométricas de fiabilidad $y$ validez.

Escala de Hipocondría del Inventario de Personalidad Multifasico de Minnesota (Hypochondriasis Scale -Hs-, Minnesota Multiphasic Personality Inventory, MMPI; Hathaway y McKinley, 1967)

La Escala de Hipocondría del MMPI (MMPIHs) está compuesta por 33 enunciados de respuesta dicotómica ("verdadero" o "falso"). Esta escala, en opinión de sus autores, permite la "evaluación del grado de preocupación anormal por las propias funciones corporales" (Hathaway y McKinley, 1967; p. 26). Pese a esta definición, algunos autores han expresado 
ciertas reservas acerca de su validez como medida de la hipocondría. Según Kellner (1987), lo que realmente estima este instrumento es la presencia de ciertos síntomas físicos, pero no permite una valoración de las preocupaciones hipocondríacas puesto que no evalúa creencias tales como la de estar sufriendo una grave enfermedad, que se mantiene a pesar de la información médica tranquilizadora, o los temores específicos, como el miedo a padecer cáncer o una enfermedad cardiaca. Como señala Edelmann (1992) si bien la escala discrimina entre pacientes hipocondrlacos y no hipocondríacos, existe un marcado solapamiento en las puntuaciones. A juicio de este autor, esta medida detecta las quejas físicas reales, pero no las discrepancias entre el estado fisico objetivo y la percepción subjetiva de éste, que es lo que caracteriza a los fenómenos hipocondríacos. Edelmann y Holdsworth (1993) han cuestionado la utilidad de la escala como medida de la hipocondria, ya que consideran que lo que en realidad evalúa es la conciencia objetiva de las sensaciones somáticas.

En cuanto a sus caracteristicas psicométricas cabe senalar que el coeficiente de fiabilidad test-retest de la MMPI-Hs es de 0,80 en una muestra normal y con un intervalo temporal entre tres dias y más de un ano (Hathaway y Mckinley, 1942); 0,81 en une muestra similar pero con un intervalo de una semana (Cottle, 1950); y 0,67 en pacientes psiquiátricos (Holzberg y Alessi, 1949). Según Hathaway y Mckinley (1967), la validez de este instrumento reside en su capacidad predictiva respecto al diagnóstico establecido por profesionales del área de la neuropsiquiatria. En una muestra española de pacientes con hipocondria (criterios DSM-III-R) la MMPI-Hs ha mostrado una aceptable consistencia intema (alfa de Cronbach $=0,76$ ) (Martínez, 1997).

Cuestionario de Conducta de Enfermedad (IIIness Behavlour Questionnaire, IBQ; Pllowsky y Spence, 1983)

EI IBQ permite medir las actitudes que indican modos desadaptativos o inapropiados de responder al propio estado de salud (Pilowsky, 1971). Es una escala compuesta por 62 items a los que se contesta según dos altemativas de respuesta: "si" o "no", y está formado por los siete factores siguientes (Pilowsky y Spence, 1983):

- Hipocondría General. Actitudes fóbicas hacia la enfermedad vinculadas a un grado elevado de ansiedad y cierto reconocimiento de que tales actitudes son inapropiadas. Incluye los items 9 , $20,21,24,29,30,32,37$ y 38.

- Convicción de Enfermedad. Creencia de estar padeciendo una enfermedad fisica, preocupación por la sintomatologia somática y rechazo de la información médica tranquilizadora. Incluye los ítems 2, 3, 7, 10, 35 y 41.

- Percepción Psicológica vs. Somática de Enfermedad. Tendencia a interpretar la enfermedad en términos psicológicos y creer en la necesidad de recibir tratamiento psicologico, frente a la tendencia a considerar que el problema es de carácter fisico y que requiere un tratamiento médico. Incluye los items $11,16,44,46$ y 57.

- Inhibición Afectiva. Dificultad para expresar a otras personas los propios sentimientos, fundamentalmente los negativos. Incluye los items 22, 36, 53, 58 y 62.

- Perturbación Afectiva. Estados afectivos de inquietud y/o aflicción. Incluye los items 12, $18,47,54$ y 59.

- Negación. Propensión a negar la existencia de problemas cotidianos y/o considerarlos consecuencias del problema físico. Incluye los Items $27,31,43,55$ y 60 .

- Irritabilidad. Estados afectivos de irritación y conflictos interpersonales. Incluye los items $4,17,51,56$ y 61.

Estos siete factores se agrupan en dos componentes de segundo orden que ofrecen una información más global de la conducta de enfermedad (Pilowsky y Spence, 1983): Estedo Afectivo (comprende los factores de Hipocondria General, Perturbación Afectiva e Irritabilidad) y Afirmación de Enfermedad (comprende los factores de Convicción de Enfermedad y Percepción Psicológica vs. Somática de Enfermedad).

EI IBQ también cuenta con un indice (Indice Whiteley) que permite explorar las actitudes hipocondriacas. El Indice Whiteley está compuesto por 14 Items que se agrupan en tres dimensiones (Pilowsky, 1967):

- Preocupación por el Cuerpo. Preocupación por sensaciones o signos fisicos. Incluye los items 1, 10, 16, 33, 34, 41 y 50. 
- Fobia a la Enfermedad. Temor a sufrir una enfermedad, "insight" que suele estar asociado a este tipo de temores y conductas de búsqueda de información tranquilizadora sobre la salud pese a admitir la ausencia de enfermedad. Incluye los items $8,21,24$ y 38 .

- Convicción de Enfermedad. Creencia firme de padecer una grave enfermedad que se acompaña de una actitud de desconfianza hacia la familia y el personal médico. Incluye los fitems 2,9 y 39.

EI IBQ también tiene una Función Discriminante que permite evaluar la presencia de una reacción de conversión. Esta función comprende los factores de Convicción de Enfermedad, Percepción Psicológica vs. Somática de Enfermedad, Inhibición Afectiva y Negación.

Pilowsky y Spence (1983) han establecido los siguientes puntos de corte para detectar a los pacientes con elevada probabilidad de manifestar conducta anormal de enfermedad:

- Convicción de Enfermedad: puntuación mayor o igual que 3 (3-6).

- Percepción Psicológica vs. Somática de Enfermedad: puntuación menor o igual que 1 ( 0 1).

- Afirmación de Enfermedad: puntuación mayor o igual que 7 (7-11).

- Indice Whiteley: puntuación mayor o igual que 8 (8-14).

- Función Discriminante: puntuación mayor o igual que 70 (70-100).

Disponemos de bastante información sobre las propiedades psicométricas del IBQ. Las correlaciones test-retest de este instrumento oscilan entre 0,67 (Inhibición Afectiva) y 0,87 (Hipocondria General y Perturbación Afectiva) entre 1 y 12 semanas después de la primera administración del cuestionario (Pilowsky y Spence, 1983). Asimismo, según estos autores, el IBQ posee una adecuada validez discriminante: los factores de Hipocondria General, Convicción de Enfermedad, Negación y Percepción Psicológica vs. Somática de Enfermedad permiten diferenciar entre pacientes de medicina general y pacientes de una clínica de dolor. Los resultados de diversos estudios han ilustrado la bondad estadistica del IBQ. Asi, por ejemplo, Zonderman, Heft y Costa (1985) y Varma, Malhotra y Chaturvedi (1986) obtuvieron estructuras factoriales del IBQ similares a la obtenida en los trabajos iniciales de Pilowsky y Spence.
No obstante, Pilowsky (1993) en una factorización posterior del IBQ encontró cinco nuevos componentes: Sensibilidad interpersonal, Reacción a la Información Médica Tranquilizadora, Impacto de la Enfermedad, Autoestima y Funciones Biologicas. Por otra parte, Chaturvedi, Bhandari, Beena y Rao (1996), respecto al factor de segundo orden del IBQ Afirmación de Enfermedad, han seńalado que el punto de corte establecido para detectar patología (puntuación mayor o igual que 7) proporciona una sensibilidad del $86 \%$, una especificidad del $83 \%$ y una tasa de detecciones positivas del 0,7537. A pesar de estos datos, el IBQ presenta varios aspectos problemáticos. Según Kellner (1987) estos serian, por una parte, la heterogeneidad de contenidos de los items que componen una misma escala y, por otra, la inclusión en algunas escalas de items relacionados con sintomatologia depresiva y percepción de sintomas somáticos. Schmidt (1994) también ha señalado algunas limitaciones psicométricas del Indice Whiteley: algunos de los items presentan saturaciones bajas en el factor (inferiores a 0,40 ); la caracterización del indice no se ajusta exactamente al contenido de los items que lo componen; y no existe información sobre su sensibilidad y especificidad. Esta última critica no podría ser mantenida actualmente puesto que ya existen datos de este tipo, tal y como acabamos de señalar al mencionar el trabajo de Chaturvedi et al. (1996).

Llor y colaboradores (Llor, Nieto, Godoy y Morales, 1991; Llor, Godoy y Nieto, 1993) han realizado una baremación, factorización y depuración de este cuestionario en muestras clínicas españolas. Asimismo, la adaptación castellana del IBQ ha mostrado una aceptable consistencia interna en una muestra de pacientes que cumplian los criterios del DSM-III-R para la hipocondria (alfa de Cronbach $=0,74$ ) (Martínez, 1997).

\section{Escalas de Actitud hacla la Enfermedad} (IIIness Attitude Scales, IAS; Kellner, 1986)

Las Escalas de Actitud hacia la Enfermedad fueron elaboradas para la valoración de la psicopatología que suele estar asociada a la hipocondria y a la conducta anormal de enfermedad (Kellner et al., 1986). En la elaboración de las IAS, Kellner intento subsanar las defi- 
ciencias que presentaban la Escala Hs del MMPI y el IBQ. Para ello, procuró que las nuevas escalas incluyeran contenidos referidos a las actitudes, miedos y creencias hipocondriacas (p. ej., la preocupación por la enfermedad o por el cuerpo) y que excluyera síntomas que también pudieran aparecer en otros trastornos ( $p$. ej., los problemas para conciliar el sueño, los sentimientos depresivos y las sensaciones somáticas) (Kellner, 1987). Las IAS incluyen 29 items agrupados en nueve subescalas de tres items cada una. Estas escalas son las siguientes (Kellner, 1986):

- Preocupación por la Enfermedad. Intranquilidad por el estado de salud actual y por la posibilidad de enfermar en un futuro (ítems 1 , 2 y 3).

- Preocupación por el Dolor. Atribución de un significado negativo a los síntomas corporales dolorosos y realización de consultas médicas vinculadas a ésta (ítems 4,5 y 6).

- Hábitos de Salud. Evitación de hábitos y alimentos que pueden perjudicar la salud y realización de conductas de comprobación del estado corporal (items 7, 8 y 9).

- Creencias Hipocondríacas. Creencias erróneas referidas a padecer una enfermedad y desconfianza ante la información médica tranquilizadora (items 10, 11 y 12).

- Tanatofobia. Temor a la muerte y a los estímulos vinculados a ella (Items 13, 14 y 15).

- Fobia a la Enfermedad. Miedos exagerados a poder estar sufriendo una enfermedad (Items 16, 17 y 18).

- Preocupación por el Cuerpo. Atención dirigida a los sintomas corporales y preocupación por ellos (items 19, 20 y 21).

- Experiencia con el Tratamiento. Frecuencia de las consultas médicas y tratamientos recibidos (items 23, 24 y 25).

- Efectos de los Sintomas. Perturbación que ocasionan los sintomas corporales en diversos ámbitos de la vida (items 27, 28 y 29).

La valoración de cada item se realiza de acuerdo con una escala Likert de 5 puntos que va desde "no" (0) hasta "casi siempre" (4), a excepción de los ítems de la escala de Experiencia con el Tratamiento cuyo rango numérico es el mismo pero varia el contenido de las alternativas de respuesta. Las IAS incluyen además dos preguntas adicionales: el ítem 22, que rastrea si existe algún trastomo fisico diag- nosticado, y el ítem 26, que recoge información sobre los tratamientos recibidos durante ol último año.

Existen tres versiones de las IAS atendiendo a las instrucciones que se dan al sujeto sobre el momento temporal al que se refieren las preocupaciones hipocondriacas (Kellner, 1987): "medida estado" (último mes), "medida rasgo" (generalmente) y "versión estándar" (sin referencia temporal).

En cuanto a las características psicométricas de las IAS, cabe decir que según Kellner (p. ej., Kellner, Abbot, Winslow y Pathak, 1987) este instrumento es fiable y tiene una adecuada validez de constructo. La correlación test-retest de las IAS en dos grupos de sujetos normales transcurridas 1 y 4 semanas respectivamente oscilaron entre 0,62 (Creencias Hipocondriacas) y 1,0 (Fobia a la Enfermedad) (Kellner, 1987). Las IAS discriminan entre los pacientes hipocondríacos y los pacientes con otros trastomos: los sujetos hipocondriacos obtienen puntuaciones elevadas (3-4) en los items de las escalas de Creencias Hipocondriacas y/o Fobia a la Enfermedad, lo que no suele ocurrir en otros pacientes (Kellner et al., 1987). La versión castellana de las IAS también ha mostrado una adecuada consistencia interna en un grupo de pacientes con hipocondria (criterios DSM-III-R) (alfa de Cronbach $=0,87$ ) (Martínez, 1997). En cuanto a la constitución de las IAS, cabe decir que Stretton, Salovey y Mayer (1993) hallaron en este instrumento una estructura factorial que compartla muchas similitudes con el análisis térico propuesto por Kellner. Ferguson y Daniel (1995) han informado que las IAS tienen una estructura de cuatro componentes: Hipocondrla General; Experiencia de Sintomas y Frecuencia de Tratamientos; Tanatofobia; y Miedo a la Enfermedad Cardiaca y Hábitos de Salud Preventivos Asociados. Por su parte, Speckens, Spinhoven, Sloekers, Bolk, et al. (1996) han encontrado una estructura bifactorial que incluye los componentes de Ansiedad por la Salud y Conducta de Enfermedad.

\section{Escala de Ampllficación Somatosensorial (Somatosensory Ampllfication Scale, SSAS; Barsky, Wyshak y Klorman, 1990) \\ Esta escala se considera un instrumento de evaluación de la hipocondria puesto que permite}


detectar la presencia de un "estilo somático amplificador", concepto que, según Barsky y colaboradores (Barsky, 1992b; Barsky y Klerman, 1983; Barsky y Wyshak, 1990; Barsky et al., 1990), permite explicar la problemática de este desorden. La SSAS fue construida con el fin de medir la sensibilidad a las sensaciones corporales benignas que son molestas, pero que no constituyen sintomas típicos de enfermedad (Barsky et al., 1990).

Este instrumento está compuesto por 10 items, a los que la persona ha de contestar señalando el grado en que cada uno de ellos "le caracteriza en general". Cada item se puntúa de acuerdo con una escala Likert de 5 puntos que oscila desde "nada en absoluto" (1) hasta "muchisimo" (5). Según un reciente trabajo (Martinez, Belloch y Botella, en revisión), la versión castellana de la SSAS consta de dos componentes:

- Amplificación de Estímulos Extermos. Tendencia a percibir de manera intensa los estímulos externos. Incluye los items 3, 4, 6, 8, 9 y 10.

- Amplificación de Estímulos Intemos. Umbral perceptivo bajo para la detección de señales interoceptivas. Incluye los items 1, 2, 5 y 7.

La SSAS posee una buena consistencia interna y fiabilidad test-retest. Así, por ejemplo, en el estudio de Barsky et al. (1990) con pacientes de medicina general, se obtuvo una consistencia interna de 0,82 y una fiabilidad test-retest de 0,79 (intervalo temporal medio de 74 dias). En opinión de estos autores, este tipo de resultados permite inferir que la amplificación somatosensorial es un constructo unitario con cierto grado de estabilidad temporal. Los resultados de la investigación de Barsky et al. (1990) han mostrado que los sujetos hipocondriacos obtienen puntuaciones superiores en la SSAS a las obtenidas en una muestra de comparación de pacientes no hipocondriacos de una clínica de medicina general, y que el nivel de amplificación correlacionó significativamente con el grado de sintomatología hipocondríaca en ambas muestras. Por otra parte, cabe decir que la versión castellana de la SSAS ha mostrado una aceptable consistencia interna en una muestra de pacientes que reunian los criterios del DSM-
III-R para la hipocondría (alfa de Cronbach = 0,77) (Martínez, 1997).

La SSAS ha recibido en los últimos años ciertas criticas. Según Kirmayer ot al. (1994) esta escala presenta los siguientes problemas: 1) es una estimación de los productos de la amplificación somática más que de los procesos cognitivos o perceptivos subyacentes; y 2) no especifica si utiliza algún criterio para diferenciar la amplificación de síntomas somáticos de otras modalidades de malestar. Asimismo, podemos añadir una limitación más (Martínez, Belloch y Botella, en revisión), esto es, problemas respecto a problemas de validez de constructo. Esta última cuestión alude a que aunque, el concepto de amplificación es concebido por Barsky como un estilo cognitivoperceptivo que incluye hipervigilancia corporal, selección y focalización de la atención sobre los sintomas corporales, $\theta$ interpretación negativa de las sensaciones físicas, la SSAS sólo evalúa los dos primeros aspectos, igniorando en su totalidad el tercero.

Escala de Preocupaciones por la Enfermedad (IIIness Concerns Scale, ICS; Wesner y Noyes, 1991)

La Escala de Preocupaciones por la Enfermedad es un cuestionario diseñado para medir la sintomatologia de la fobia a la enfermedad tanto en sus manifestaciones cognitivas (p. ej., preocupación, rumiación) como en las comportamentales (p. ej., evitación, búsqueda de información tranquilizadora). Consta de 18 enunciados (algunos de ellos pertenecientes al IBQ) cada uno de los cuales es valorado en una escala Likert de 5 puntos que comprende desde "nada en absoluto" (0) hasta "muchísimo" (4). Los únicos datos que disponemos hasta el momento acerca de la fiabilidad y validez de la adaptación castellana de esta medida proceden del trabajo de Jiménez, Castaneiras, Martínez, Ayllón y Belloch (1997). Los datos de este estudio, realizado sobre una muestra de 300 estudiantes universitarios, revelaron una adecuada consistencia interna (alfa de Cronbach = 0,88 ) y validez discriminante. Además, se observó que esta medida presenta una estructura integrada por dos factores: Rumiación/Preocupación, y Evitación/Aprensión Ansiosa. 
Cuestionario de Ansiedad Cardfaca (Cardiac Anxiety Questionnaire, CAQ; Eifent, Seville, Brown, Anthony y Barlow, 1992)

El Cuestionario de Ansiedad Cardiaca es un ejemplo de los instrumentos que se están desarrollando en los últimos años para la valoración de las preocupaciones de carácter hipocondriaco centradas en enfermedades especificas (p. ej., enfermedades coronarias, cancer, SIDA). Este instrumento evalúa las caracteristicas básicas de la ansiedad por el estado del corazón. EI CAQ está compuesto por 16 ítems a los que el sujeto ha de contestar, de acuerdo con una escala Likert de 5 puntos (entre "nunca" -0- y "siempre" -4-), con qué frecuencia tiene una serie de pensamientos y realiza ciertas conductas. Los items se agrupan en cuatro dimensiones: 1) Convicción de Enfermedad Cardiaca/Conciencia Cardiaca; 2) Conducta Cardioprotectora; 3) Búsqueda de Ayuda Médica; y 4) Hiperarousal. Las subescalas de esta medida han mostrado una adecuada consistencia interna (alfa de Cronbach de 0,87, 0,79, 0,84 y 0,73, respectivamente; Eifert ot al., 1992; citado en Eifert y Forsyth, 1996).

\section{PERSPECTIVAS FUTURAS}

De acuerdo con la revisión realizada sobre la evaluación de la hipocondria, podemos afirmar que en los últimos años se han conseguido importantes avances en la mejora de las estrategias para rastrear la presencia de esta clase de problemas. Asi, por ejemplo, se han detallado las características formales y de contenido que debe tener la entrevista de evaluación, y se han elaborado cuestionarios de autoinforme cada vez más específicos que permiten explorar con mayor precisión los diversos aspectos clínicos de este trastorno. Pese a estos avances, todavla existen bastantes aspectos que merecen un análisis detallado, como por ejemplo, el examen en profundidad y la valoración de los procesos cog-nitivos que subyacen a los fenómenos hipocondriacos, o la existencia de posibles subtipos o modalidades de hipocondría.

Respecto a los procesos cognitivos que subyacen a la hipocondria, en la última década se vienen realizando algunos intentos notables por examinar la presencia de ciertos sesgos que probablemente operan en distintos niveles o ámbitos de procesamiento de los pacientes que presentan este trastomo. Asi, por ejemplo, algunos estudios utilizando cuestionarios de autoinforme, han explorado los sesgos interpretativos que presentan los hipocondriacos mediante el análisis de las atribuciones emóneas que realizan sobre sensaciones corporales ambiguas (p. ej., Salkovskis, 1990; Hitchcock y Mathews, 1992; Martínez, Belloch y Botella, 1995). En otros estudios se han analizado los sesgos mnésicos de los pacientes hipocondríacos, mediante tareas experimentales que exploran la propensión de éstos a recordar hechos relacionados con su preocupación ( $p$. ej., Durso, Reardon, Shore y Delys, 1991). En cuanto a los sesgos atencionales, cabe senalar que a pesar de ser, junto con los sesgos interpretativos, una de las distorsiones cognitivas más estrechamente vinculada a la hipocondria, sólo algunos autores (p. ej., Cioffi, 1991; Pauli, Schwenzer, Brody, Rau y Birbaumen, 1993; Schmidt, Wolfs-Takens, Oosterlaan y van den Hout, 1994) se han ocupado de su estudio.

Una de las estrategias experimentales más utilizadas para explorar la presencia de sesgos atencionales es la versión "emocional" de la Tarea de Stroop. Este procedimiento incluye palabras cuyo contenido es emocionalmente significativo para un sujeto y palabras con un contenido neutral, escritas en diferentes colores. La instrucción que se le da al individuo consiste en pedirle que nombre lo más rápidamente posible el color en que están escritas las palabras, ignorando su significado. La í́gica que subyace a la técnica reside en que el sesgo atencional hacia ciertos temas o contenidos se reflejará en una mayor lentitud al completar la tarea cuando el contenido de las palabras es emocionalmente significativo para el individuo, que si se trata de palabras sin significado emocional. Es decir, el material estimular (contenido o significado de las palabras) atraerá de forma involuntaria la atención, y como consecuencia la realización de la tarea (nombrar los colores) empeorará. Asi pues, el significado de la palabra y el color en el que está escrita, "compiten" para captar los recursos atencionales del sujeto (McNally, Riemann y Kim, 1990).

Desde que Mathews y MacLeod (1985) empezaran a utilizar en el ámbito de los trastornos emocionales el Paradigma Stroop para la evaluación de los sesgos atencionales, se han realizado muchos trabajos replicando los 
resultados obtenidos sobre la existencia de este tipo de sesgos en diversas condiciones clínicas -depresión, trastorno por an-siedad generalizada, trastorno por angustia-; los resultados más consistentes se han encontrado, precisamente, en el ámbito de los trastornos por ansiedad, constatándose el "efecto Stropp" cuando el contenido de las palabras-estímulo hace referencia a amenazas. Sin embargo, no existen estudios que hayan investigado la atención diferencial a los estímulos amenazantes en la hipocondría a través de este procedimiento. De hecho, el único estudio, que tengamos constancia, que ha utilizado la Tarea de Stroop en muestras de pacientes con hipocondría es el realizado por las autoras de este trabajo (Martinez, 1997; Martinez y Belloch y Botella, en preparación).

Creemos que el desarrollo, la validación y la utilización de procedimientos de evaluación cognitiva, pueden ser de enorme relevancia para optimizar nuestra comprensión de la hipocondrla y mejorar el modo de combatirla. Así, por ejemplo, este tipo de procedimientos puede contribuir a verificar las teorias cognitivas explicativas sobre el desarrollo y mantenimiento de este desorden, a detectar a los pacientes hipocondríacos en los que predominen las tendencias atencionales problemáticas, a disenar estrategias de intervención destinadas a corregir tales tendencias, y a valorar el grado de cambio logrado en los sesgos cognitivos de los pacientes que reciben tratamiento psicologico.

En cuanto a la segunda área de interés en la evaluación de la hipocondría, esto es, los subtipos, cabe decir que desde hace algunos anos se viene apuntado la posibilidad de que la hipocondrla no constituya una entidad única sino que comprenda diversas modalidades, cada una de ellas con determinadas características fenomenológicas, que requieren instrumentos de medida especificos y estrategias de intervención diferentes. En un estudio reciente las autoras de este trabajo hemos esbozado tres hipotéticas modalidades de hipocondria (Martínez, 1997). Una primera clase de hipocondría sería aquella caracterizada por la creencia anómala en la existencia de una enfermedad orgánica. El ámbito más afectado sería el del pensamiento, pudiendo manifestarse en cualquiera de las formas incluidas en el continuo que se extiende desde la idea sobrevalorada llegando hasta el delirio.
Los procesos cognitivos más relevantes serian de carácter controlado, y los sesgos cognitivos se constatarian principalmente a nivel de productos. El tratamiento se centraría en el empleo de la prueba de realidad y del desafio verbal.

Un segundo subtipo de hipocondría sería aquel que presenta principalmente un componente de temor a la enfermedad. El problema más relevante sería la existencia de trastornos atencionales (focalización de la atención e hipervigilancia). Los procesos cognitivos implicados serian esencialmente automáticos. EI núcleo psicopatológico de esta condición sería la codificación selectiva de las amenazas a la salud. El tratamiento incluirla entre sus elementos más importantes el entrenamiento atencional, la exposición a los estímulos amenazantes y la prevención de respuesta de las conductas problemáticas.

Una tercera clase de hipocondría sería aquella en la que el problema nuclear se circunscribiria al ámbito de la percepción. Este trastorno podria entenderse como una condición situada en cualquiera de los puntos comprendidos entre los extremos delimitados por la distorsión perceptiva (anomalía en la percepción de la intensidad de los estimulos -estilo somático amplificador-) y el engaño perceptivo (alucinación somática). En este modo de conceptualizar la hipocondria estarian involucrados tanto procesos cognitivos controlados como automáticos. Los elementos psicopatológicos nucleares incluirían características tanto de la creencia de enfermedad como del miedo a la enfermedad. Asimismo, la intervención englobaría algunos de los elementos propuestos para los dos subtipos anteriores.

La posible existencia de estos tres subtipos de hipocondria, apunta a la necesidad de disenar instrumentos de medida que permitan la detección correcta de cada uno de ellos. Posiblemente, en el caso de la creencia de enfermedad los instrumentos de medida al ir destinados a valorar productos cognitivos, tendrian formato de cuestionarios estandarizados de auto-informe, similares a los descritos en este trabajo. Para el miedo a la enfermedad y puesto que se trataría de rastrear la codificación selectiva de las amenazas, el método más idóneo serian las pruebas atencionales (p. ej., la Tarea de Stroop). En el caso de la hipocondria entendida como estilo somático amplificador se recurriría a ambos 
tipos de instrumentos. Asimismo, y si se hallan datos consistentes con este planteamiento, cabría proponer para cada subtipo de hipocondría modos de evaluar el cambio terapéutico conseguido acordes con los elementos psicopatológicos que se pretende modificar.

En definitiva, la evaluación psicologica de la hipocondría está absolutamente determinada, como sucede para cualquier otro trastorno mental, por la caracterización psicopatológica y clínica que se realice. En los últimos años se ha avanzado bastante en este aspecto, y por lo tanto en el desarrollo de instrumentos fiables y válidos para la evaluación psicológica del trastorno. Pero seguramente la carrera no ha hecho más que comenzar y todavla estamos bastante lejos de comprender adecuadamente la naturaleza de la hipocondria, su relación y diferencia con otras psicopatologias, o su curso clínico.

\section{Agradecimientos}

Este estudio ha sido financiado a través de una Beca de Investigación concedida por la Conselleria d'Educació i Ciencia de la Generalitat Valenciana a la primera de las autoras, para llevar a cabo una investigación más extensa de la cual forma parte este trabajo.

\section{REFERENCIAS BIBLIOGRAFICAS}

American Psychiatric Association (1980). Diagnostic and Statistical Manual of Mental disorders (3* ed.). Washington, DC, APA (versión española: Barcelona, Masson, 1981).

American Psychiatric Association (1987). Diagnostic and Statistical Manual of Mental disorders ( $3^{\circ}$ ed. rev.). Washington, DC. APA (versión española: Barcelona, Masson, 1988).

American Psychiatric Association (1994). Diagnostic and Statistical Manual of Mental disorders (4" ed.). Washington, DC, APA. (versión española: Barcelona, Masson, 1995).

Avia, M. D. (1993). Hipocondria. Barcelona, Martínez Roca.

Barsky, A. J. (1992a). Hypochondriasis and obsessive-compulsive disorder. Psychiatric Clinics of North America, 15 (4), 791-801.

Barsky, A. J. (1992b). Amplification, somatization, and the somatoform disorders. Psychosomatics, 33, 28-34.

Barsky, A. J., Cleary, P. D., Wyshak, G., Spitzer, R., Williams, J., y Klerman, G. (1992). A structured diagnostic interview for hypochondriasis: a proposed criterion standard. Journal of Nervous and Mental Diseases, 180, 20-27.

Barsky, A. J., Wyshak, G., y Klerman, G. L. (1986). Hypochondriasis: an evaluation of the DSM-III criteria in medical outpatients. Archives of General Psychiatry, 43, 493-500.

Barsky, A. J., Wyshak, G., y Klerman, G. L. (1990). The somatosensory amplification scale and its relationship to hypochondriasis. Journal of Psychiatric Research, 24, 323-334.

Barsky, A. J., y Klerman, G. L. (1983). Overview: hypochondriasis, bodily complaints, and somatic styles. American Journal of Psychiatry, 140, 273-283.

Barsky, A. J., y Wyshak, G. (1990). Hypochondriasis and somatosensory amplification. British Joumal of Psychiatry, 157, 404-409.

Cioffi, D. (1991). Sensory awareness versus sensory impression: affect and attention interact to produce somatic meaning. Cognition and Emotion, 5 (4), 275-294.

Clark, L. A., Watson, D., y Reynolds, S. (1995). Diagnosis and classification of psychopathology: challenges to the current system and future directions. Annual Review of Psychology. 46. 121-153.

Costa, P. T., y McCrae, R. R. (1985). Hypochondriasis, neuroticism, and aging: when are somatic complaints unfounded? American Psychologist, 40 (1), 19-28.

Cottle, W. C. (1950). Card versus booklet forms of the MMPI. Joumal of Applied Psychology, 34, 255-259.

Chaturvedi, S. K., Bhandari, S., Beena, M. B., y Rao, S. (1996). Screening for abnormal illness behaviour. Psychopathology, 29, 325-330.

Chorot, P., y Martinez, M. P. (1995). Trastomos somatoformes. En A. Belloch, B. Sandin, y F. Ramos (dirs.), Manual de Psicopatologla Nol. 2). Madrid, McGraw-Hill.

Dubovsky, S. L. (1988). Concise Guide to Clinical Psychiatry. Washington, DC, American Psychiatric Press.

Durso, F. T., Reardon, R., Shore, W. J., y Delys, S. $M$. (1991). Memory processes and hypochondriacal tendencies. Joumal of Nervous and Mental Diseases, 179 (5), 279-283.

Edelmann, R. J. (1992). Anxiety: Theory, Research and Intervention in Clinical and Health Psychology. Chichester, J. Wiley \& Sons.

Edelmann, R. J., y Holdsworth, S. (1993). The Minnesota multiphasic personality inventory hypochondriasis scale: its relation to bodily awareness and irrational beliefs. Personality and Individual Differences, 14, 369-370.

Eifert, G. H., Seville, J. L., Brown, T. A., Anthony, M., y Barlow, D. H. (1992). Cardiac anxiety 
questionnaire: a brief measure to assess heartfocused anxiety and cardiophobic behaviours. Trabajo presentado en la $26^{*}$ Conferencia Anual de AABT, Boston, MA.

Eifert, G. H., y Forsyth, J. P. (1996). Heart-focused and general illness fears in relation to parental medical history and separation experiences. Behaviour Research and Therapy, 34 (9), 735739.

Fava, G. A., Grandi, S., Michelacci, L., Saviotti, F. M., Conti, S., Bovicelli, L., Trombini, G., y Orlandi, C. (1990). Hypochondriacal fears and beliefs in pregnancy. Acta Psychiatrica Scandinavica, 82 (1), 70-72.

Ferguson, E., y Daniel, E. (1995). The Illness Attitudes Scale (IAS): a psychometric evaluation on a non-clinical population. Personality and Individual Differences, 18 (4), 463-469.

Ford, C. V. (1983). The Somatizing Disorders: IIIness as a Way of Live. New York, Elsevier.

Ford, C. V., y Parker, P. E. (1990). Somatoform disorders. En M. E. Thase, B. A. Edelstein, y M. Hersen (dirs.), Handbook of Outpatient Treatment of Adults.

Hathaway, S. R., y Mckinley, J. C. (1967). Minnesota Multiphasic Personality Inventory. Menual Revised 1967. New York, The Psychological Corporation (versión española: Madrid, TEA, 1988).

Hathaway, S. R., y Mckinley, J. C. (1942). A multiphasic personality schedule (Minnesota): III. The measurement of symptomatic depression. Joumal of Psychology, 14, 73-84.

Hitchcock, P. B, y Mathews, A. (1992). Interpretation of bodily symptoms in hypochondriasis. Behaviour Research and Therapy, 30 (3), 223234.

Holzberg, J. D., y Alessi, S. (1949). Reliability of the shortened MMPI. Journal of Consulting Psychology, 13, 288-292.

Janca, A., Üstün, T. B., y van Drimmelen, J. (1992). The ICD-10 Classification of Mental and Behavioural Disorders: Symptoms Checklist, Version 1.0. Ginebra, WHO.

Jiménez, M. A., Castaneiras, C., Martínez, M. P., Ayllón, E., y Belloch, A. (1997). Validación de la Escala de Preocupaciones por la Enfermedad. Trabajo presentado en el I Congreso de la Asociación Española de Psicología Clínica y Psicopatologla. Madrid, 3-5 de Abril.

Kellner, R. (1986). Somatization and Hypochondriasis. Nueva York, Praeger.

Kellner, R. (1987). Abridged Manual of the Illness Attitude Scales. Departamento de Psiquiatría, Universidad de Nuevo México, USA.

Kellner, R., Abbott, P., Winslow, W. W., y Pathak, D. (1987). Fears, beliefs, and attitudes in DSM-
III hypochondriasis. Joumal of Nervous and Mental Disease, 175, 20-25.

Keliner, R., Wiggins, R. G., y Pathak, D. (1986). Hypochondriacal fears and beliefs in medical and law students. Archives of General Psychiatry, 43, 487-489.

Kirmayer, L. J., Robbins, J. M., y Paris, J. (1994). Somatoform disorders: personality and the social matrix of somatic distress. Journal of Abnormal Psychology, 103 (1), 125-136.

Lemos, S. (1994). Clasificación y diagnóstico en psicopatologia. En A. Belloch, B. Sandín, y F. Ramos (dirs.), Manual de Psicopatología Nol. 1). Madrid, McGraw-Hill.

Lorr, M. (1986). Clasifying psychotics: dimensional and categorial approaches. En T. Millon, y G. L. Klerman (dirs.), Contemporary Directions in Psychopathology. New York, Guilford Press.

Llor, B., Godoy, C., y Nieto, J. (1993). Factorización y depuración del cuestionario IBQ en población clínica española. Anales de Psiquiatría, 9, 209-213.

Llor, B., Nieto, J., Godoy, C., y Morales, J. M. (1991). Baremo del cuestionario IBQ en población clínica española. Actas Luso-Españolas de Neurologia, Psiquiatria y Clencias Afines, 19, 263-267.

Marshall, W. R. (1987). Chronic somatizers. En C. S. Rogers, y J. D. McCue (dirs.), Managing Chronic Disease. Medical Economics Books.

Martínez, M. P. (1997). Nuevas Aproximaciones Cognitivas a la Conceptualización y al Tratamiento de le Hipocondría. Tesis Doctoral. Universidad de Valencia.

Martínez, M. P., Belloch, A. y Botella, C. (en preparación). Procesamiento de la información de amenaza fisica en la hipocondrla: un estudio exploratorio utilizando el Paradigma de Stroop.

Martinez, M. P., Belloch, A., y Botella, C. (en revisión). Somatosensory amplificacion in hypochondriasis and panic disorder.

Martínez, M. P., Belloch, A., y Botella, C. (1995). Sesgos en la interpretación de sensaciones corporales ambiguas on la hipocondría. Revista Argentina de Clínica Psicológica, 4 (3), 259-276.

Mathews, A., y MacLeod, C. (1985). Selective processing of threat cues in anxiety states. Behaviour Research and Therapy, 23, 563-569.

McNally, R. J., Riemann, B. C., y Kim, E. (1990). Selective processing of threat cues in panic disorder. Behaviour Research and Therapy, 28 (5), 407-412.

Millon, T. (1991). Classification in psychopathology: rationale, alternatives, and standards. Joumal of Abnormal Psychology, 100 (3), 245-261. 
Pauli, P., Schwenzer, M., Brody, S., Rau, H., y Birbaumer, N. (1993). Hypochondriacal attitudes, pain sensitivity, and attentional bias. Journal of Psychosomatic Research, 37 (7), 745-752.

Pilowsky, I. (1967). Dimensions of hypochondriasis. British Journal of Psychiatry, 113, 88-93.

Pilowsky, I. (1971). The diagnosis of abnormal illness behaviour. Australia and New Zealand Journal of Psychiatry, 5, 136-138.

Pilowsky, I. (1993). Dimensions of illness behaviour as measured by the illness behaviour questionnaire: a replication study. Journal of Psychosomatic Research, 37, 5362.

Pilowsky, I., y Spence, N. D. (1983). Manual for the IIIness Behaviour Questionnaire. Departamento de Psiquiatría, Universidad de Adelaida, Australia.

Salkovskis, P. M. (1989). Somatic problems. En K. Hawton, P. M. Salkovskis, J. Kirk, y D. M. Clark (dirs.), Cognitive-Behaviour Therapy for Psychiatric Problems: A Practical Guide (pp. 235-276). Oxford, Oxford University Press.

Salkovskis, P. M. (1990). The Nature of and Interaction Between Cognitive and Physiological Factors in Panic Attacks and their Treatment. Tesis Doctoral no publicada.

Salkovskis, P. M., y Clark, D. M. (1993). Panic disorder and hypochondriasis. Advances in Behaviour Research and Therapy, 15, 23-48.

Salkovskis, P. M., y Warwick, H. M. C. (1986). Morbid preoccupations, health anxiety and reassurance: a cognitive-behavioural approach to hypochondriasis. Behaviour Research and Therapy, 24 (5), 597-602.

Schmidt, A. J. M. (1994). Bottlenecks in the diagnosis of hypochondriasis. Comprehensive Psychiatry, 35 (4), 306-315.

Schmidt, A. J. M., Wolfs-Takens, D. J., Oosterlaan, J., y van den Hout, M. A. (1994). Psychological mechanisms in hypochondriasis: attentioninduced physical symptoms without sensory stimulation. Psychotherapy and Psychosomatics, 61, 117-120.

Speckens, A. E. M., Spinhoven, P., Sloekers, P. P. A., Bolk, J. H., et al. (1996). A validation study of the Whiteley Index, the Illness Attitude Scales, and the Somatosensory Amplification Scale in general medical and general practice patients. Journal of Psychosomatic Research, 40 (1), 95104.

Spitzer, R. L., Williams, J. B. W., Gibbon, M., y First, M. B. (1990). User's Guide for the Structured Clinical Interview for DSM-III-R. Washington, DC, American Psychiatric Press (versión española: Barcelona, Trajecte, 1993).

Stretton, M. S., Salovey, P., y Mayer, J. D. (1993). Assessing health concerns. Imagination, Cognition and Personality, 12 (2), 115-137.

Varma, V. K., Malhotra, A. K., y Chaturvedi, S. K. (1986). Illness Behaviour Questionnaire (IBQ): Translation and adaptation in India. Indian Joumal of Psychiatry, 28 (1), 41-46.

Warwick, H. M. C. (1995). Assessment of hypochondriasis. Behaviour Research and Therapy, 33 (7), 845-853.

Wesner, R. B., y Noyes, R. (1991). Imipramine an effective treatment for illness phobia. Joumal of Affective Disorders, 22, 43-48.

Woody, S., y Rachman, S. (1994). Generalized anxiety disorder (GAD) as an unsuccessful search for safety. Clinical Psychology Review, 14 (8), 743-753.

World Health Organization (1992). Classification of Mental and Behavioural Diseases: Clinical Descriptions and Diagnostic Guidelines (10* ed.). Ginebra, WHO (versión espanola: Madrid, Meditor, 1992).

Zonderman, A. B., Heft, M. W., y Costa, P. T. (1985). Does the abnormal illness behavior questionnaire measure abnormal illness behavior?. Health Psychology, 4, 425-436. 